\title{
Prurido Crónico: Fisiopatologia, Classificação Clínica, Diagnóstico e Tratamento
}

\author{
Manuel P. Pereira', Sonja Ständer ${ }^{1}$ \\ 'Centro de Prurido Crónico, Departamento de Dermatologia, Hospital Universitário de Münster, Alemanha
}

RESUMO - Prurido com seis ou mais semanas de duração é considerado crónico. Pode ter como origem causas dermatológicas, sistémicas, neurológicas, psicossomáticas ou psiquiátricas ou advir de uma combinação de vários factores. Devido a processos de cronificação, nomeadamente sensibilização neuronal periférica e central, o prurido pode persistir apresar do tratamento da causa subjacente. Além disso, prurido crónico leva frequentemente a doenças reativas, como depressão, ansiedade ou distúrbios de sono, tendo como consequência um substancial decréscimo da qualidade de vida. Devido à multidimensionalidade do prurido crónico, esta condição representa um desafio importante para o médico assistente. A classificação do doente com prurido crónico de acordo com a apresentação clínica facilita a orientação dos procedimentos de diagnóstico necessários bem como ajuda a estabelecer uma estratégia terapêutica. A nível terapêutico uma abordagem por etapas é recomendada. Primeiramente devem-se iniciar medidas básicas como a aplicação de emolientes para a xerose cutânea, corticoesteroides tópicos para pele inflamada ou com escoriações bem como o uso de medicamentos anti-histamínicos. Caso a origem do prurido crónico seja conhecida, deve-se proceder, se possível, ao tratamento da causa subjacente. Se a causa permanecer desconhecida ou não for passível de tratamento, uma terapia sintomática multimodal com agentes tópicos e sistémicos é frequentemente necessária. Com o aumento do conhecimento dos mecanismos patofisiológicos subjacentes ao prurido crónico, novos fármacos tem sido desenvolvidos mostrando resultados promissores.

PALAVRAS-CHAVE - Doença Crónica; Prurido/classificação; Prurido/diagnóstico; Prurido/fisiopatologia; Prurido/tratamento.

\section{Chronic Pruritus: Pathophysiology, Clinical Classification, Diagnostic and Treatment}

ABSTRACT - Pruritus persisting for six weeks or longer is considered chronic. It may arise from dermatological, systemic, neurological, psychosomatic or psychiatric conditions or result from a combination of several factors. Due to chronicity processes, such as peripheral and central sensitization, pruritus may persist even after treatment of the underlying cause. Additionally chronic pruritus constitutes often a high burden for the affected patients, who frequently develop associated conditions, such as anxiety, depression or sleep disorders. Owing to the multiple dimensions of chronic pruritus, it presents a diagnostic and therapeutic challenge to the attending physician. The categorization of the condition according to the clinical presentation helps directing the diagnostic and treatment efforts. Therapeutically a step-wise approach should be undertaken. First basic measures, such as the use of emollients for dry skin, topical steroids for inflamed or excoriated skin and antihistamines should be initiated. If the origin underlying the chronic pruritus is found, a causal therapy should be attempted. If no cause is found or a causal treatment is not possible, a symptomatic multimodal therapy with topical and systemic agents is often necessary. With increasing knowledge of the pathophysiological mechanisms underlying chronic pruritus, novel drugs with promising effects are being developed.

KEYWORDS - Chronic Disease; Pruritus/classification; Pruritus/diagnosis; Pruritus/therapy; Pruritus/physiopathology.

\section{INTRODUÇÃO}

Prurido, descrito como uma sensação desagradável levando à necessidade de coçar para obter alívio, é o sintoma mais frequente em dermatologia, sendo altamente incapacitante para quem dele padece. Na sua forma aguda, representa um mecanismo de defesa, ocorrendo por exemplo em resposta a reações alérgicas a determinados medicamentos ou após contacto com certas plantas ou animais.
Correspondência: Manuel P. Pereira

Centro de Prurido Crónico (KCP) - Departamento de Dermatologia Hospital Universitário de Münster

Von-Esmarch-Str. 58 - 48149 Münster

E-mail: ManuelPedro.Pereira@ukmuenster.de

DOI: https://dx.doi.org/10.29021/spdv.75.4.793
Recebido/Received

10 Agosto/August 2017

Aceile/Accepted

14 Agosto/August 2017 


\section{Educação Médica Contínua}

Todavia, ao tornar-se crónico, isto é quando persiste por um período igual ou superior a seis semanas, este sintoma perde a sua função protetora tornando-se patológico. Estima-se que o prurido crónico (PC) seja altamente prevalente. Um estudo realizado na Alemanha analisando a população ativa revelou que cerca de $17 \%$ dos inquiridos sofriam de $\mathrm{PC}^{l}$ enquanto um outro estudo mais recente tendo a população geral como alvo indicou uma prevalência de $22 \%$ de PC ao longo da vida. ${ }^{2}$ Particularmente em clínicas dermatológicas o prurido é um sintoma descrito frequentemente, mesmo quando os doentes não se queixam ativamente dele. ${ }^{3}$ Assim, o PC foi considerado um dos 50 sintomas interdisciplinares causando maior morbilidade. ${ }^{4}$

A abordagem ao doente com PC torna-se um desafio para os dermatologistas, devido à complexidade desta doença. ${ }^{5}$ Diversas causas, dermatológicas ou não, podem estar subjacentes ao PC, exigindo um grande esforço diagnóstico e o desenvolvimento de um plano terapêutico individual. Ademais, distúrbios reativos como estados depressivos, comprometimento do sono ou a reclusão social, estão frequentemente associados ao PC e merecem igualmente a atenção do médico assistente. Este artigo de educação médica contínua tem como objectivo fornecer um resumo das noções atuais da fisiopatologia, etiologia, classificação clínica, procedimentos de diagnóstico e abordagem terapêutica relativos ao PC.

\section{FISIOPATOLOGIA}

Com o crescente interesse da comunidade científica nos mecanismos fisiopatológicos subjacentes ao prurido, tem-se observado um aumento substancial de conhecimento nesta área. Em particular, foram já identificados diversas substâncias pruritogénicas envolvidas na ativação de receptores de fibras nervosas periféricas. Incluem-se neste grupo vários mediadores como histamina, diversas interleucinas (IL6, IL8, IL31), leucotrienos, fator de necrose tumoral alfa (TNFa), e factor de crescimento neuronal, entre outros. Estas substâncias são libertadas após dano tecidual por queratinócitos, fibroblastos, mastócitos e macrófagos, entre outras, ativando fibras nervosas periféricas. ${ }^{6,7}$

Na transmissão do prurido a nível periférico, as fibras nervosas não mielinizadas de tipo $C$ desempenham um papel essencial. Estas dividem-se em dois grupos. Fibras insensíveis a estímulos mecânicos $\left(\mathrm{C}_{\mathrm{Ml}}\right)$ são ativadas por histamina e exprimem receptores $\mathrm{H} 1$ e $\mathrm{H} 4$, bem como TRPV1. Recentemente foram descobertas fibras nervosas tipo C sensíveis a estímulos mecânicos e temperaturas elevadas $\left(C_{M H}\right)$, que também desempenham um papel importante na transmissão do prurido. Estas são insensíveis a histamina, sendo ativadas por cowhage, uma substância encontrada na planta tropical mucuna pruriens, e exprimem diversos receptores como TRPV1 e PAR-2. ${ }^{8}$ Também as fibras nervosas do tipo $A \delta$ estão envolvidas na transmissão periférica do prurido.

A nível central, diferentes grupos de neurónios têm sido associados à transmissão do prurido. Neurónios exprimindo receptores de neuroquina 1 estão ligados à transmissão da dor e prurido, enquanto neurónios exprimindo receptores do péptido libertador de gastrina (GRPR) estão especificamente envolvidos na transmissão do prurido. Também interneurónios inibidores (Bhlhb5+) têm a capacidade de suprimir prurido a nível espinal. 6 Através do trato espinotalâmico, os impulsos nervosos são transportados até ao tálamo, e desta estrutura, são distribuídos para diversos centros cerebrais. Tanto centros corticais como subcorticais participam no processamento central do prurido, incluindo centros de processamento de informação sensorial, afectiva e atividade motora. Em parte estes centros correspondem aos centros ativados em estados de dor. Não existe no entanto uma matriz de ativação cerebral específica do prurido. ${ }^{6}$

A distinção entre prurido agudo e crónico tem relevância prognóstica e terapêutica. Ao fim de uma persistência de pelo menos seis semanas deste sintoma, estima-se que ocorram fenómenos de cronificação, incluindo processos de sensibilização nervosa periférica e central, contribuindo para a perpetuação do sintoma e, assim, para a cronificação do estado patológico. Desta forma, PC torna-se complexo de tratar, sendo que se deve ter em conta estes processos de cronificação ao desenvolver um plano terapêutico.

\section{CLASSIFICAÇÃO CLÍNICA E ETIOLÓGICA}

Com o intuito de categorizar o PC assim como uniformizar a abordagem ao doente com PC, o fórum internacional para o estudo do prurido (International Forum for the Study of Itch; IFSI) desenvolveu uma classificação clínica simples. Nesta classificação, os doentes com PC são divididos em três categorias de acordo com a sua apresentação clínica: i) doentes com doença cutânea inflamatória (grupo IFSI I); ii) doentes sem alteração cutânea (grupo IFSI II); iii) doentes com lesões crónicas associadas a manipulação prolongada, nomeadamente prurigo crónico e líquen simples crónico (grupo IFSI III; Fig. 1). ${ }^{9}$

Outra classificação relevante na abordagem ao doente com PC, igualmente desenvolvida pela IFSI, é a classificação etiológica, isto é o agrupamento do doente com base na causa subjacente ao PC. Doenças dermatológicas (i), sistémicas (ii), neurológicas (iii) e psiquiátricas ou somatoformes (iv) podem estar na origem do PC. Não raras vezes a presença concomitante de múltiplas enfermidades passíveis de causar prurido (PC multifactorial) é identificada num doente ( $v$ ) enquanto, nalguns casos, a origem permanece desconhecida (vi). Estas possíveis causas de PC correspondem assim aos seis grupos etiológicos previstos nesta classificação. ${ }^{9}$ Enfermidades passíveis de induzir PC são apresentadas na Tabela 1 divididas de acordo com o grupo etiológico correspondente. A classificação do doente com PC na respectiva categoria clínica e etiológica facilita a orientação dos procedimentos de diagnóstico necessários e auxilia no desenvolvimento de uma estratégia terapêutica em cada caso específico. 


\section{Educação Médica Contínua}
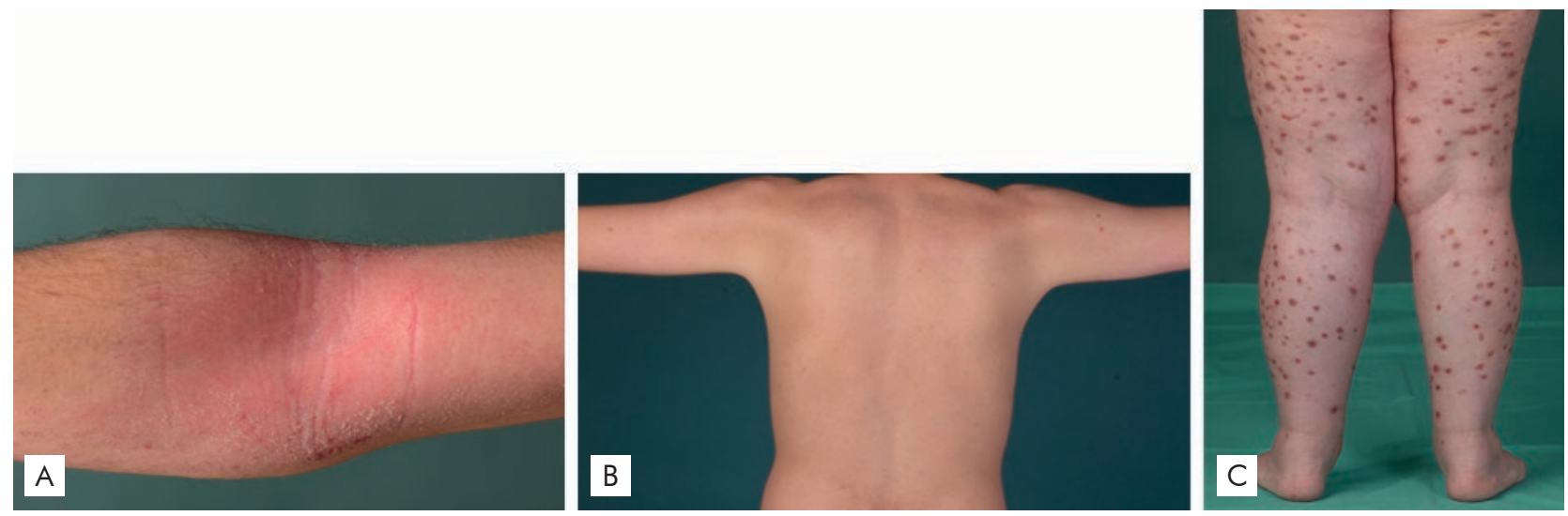

Figura 1 - Documentação fotográfica exemplar de doentes com prurido crónico agrupados de acordo com a classificação clínica proposta pelo IFSI. A) Doente de 31 anos do sexo masculino com dermatite atópica (grupo IFSI I). B) Doente de 24 anos do sexo masculino com prurido colestático devido a colangite esclerosante primária (grupo IFSI II). C) Doente de 62 anos do sexo feminino com disposição atópica apresentando lesões de prurigo crónico (grupo IFSI III). IFSI: International Forum for the Study of Itch.

\section{DIAGNÓSTICO}

Essencial na investigação da causa subjacente ao PC é a obtenção de uma história clínica minuciosa e de um exame físico completo. A anamnese deve incluir detalhes acerca do prurido em si (localização, qualidade, intensidade, duração, carácter episódico ou contínuo, factores agravantes e aliviantes) bem como sobre doenças concomitantes e medicação atual. Uma informação de grande relevância é a duração da doença prurítica. No caso de PC com duração inferior a uma ano deve-se excluir causa maligna, como p. ex. doença de Hodgkin, sobretudo caso se observem outros sinais como perda de peso ou suores noturnos. Também a relação temporal do início do sintoma com um evento relevante (p. ex. nova medicação ou hospitalização) podem revelar pistas quanto à etiologia do PC. Deve-se ainda perguntar se aquando do aparecimento do prurido se observavam alterações cutâneas, ajudando assim a diferenciar entre causas dermatológicas ou sistémicas. Caso outros membros da família padeçam igualmente de prurido, deve-se excluir uma causa infecciosa. Prurido induzido mecanicamente é comum na dermatite atópica, urticária, mastocitose e em doença colestática, enquanto que o prurido aquagénico aponta para uma possível doença hematológica subjacente. A avaliação da intensidade do prurido ao longo das consultas de seguimento permite documentar a evolução do sintoma bem como a resposta à terapia. Finalmente, deve-se também questionar o doente acerca de condições reativas, como a depressão, ansiedade ou distúrbios de sono, bem como sobre um possível prejuízo da qualidade de vida.

Questionários estandardizados acerca das características do prurido podem ser preenchidos pelos doentes na sala de espera antes da consulta, permitindo ao médico obter uma vasta informação sobre a condição que aflige o doente em pouco tempo. ${ }^{10,11}$

No exame objectivo é de particular relevância a identificação de eventuais afeções cutâneas primárias ou secundárias bem como a categorização do doente de acordo com a sua classificação clínica (ver subcapítulo Classificação Clínica e Etiológica).

Tabela 1 - Possíveis causas de prurido crónico de acordo com a classificação etiológica proposta pelo IFSI.

\begin{tabular}{l|l}
\multicolumn{1}{c|}{ GRUPO ETIOLÓGICO } & \multicolumn{1}{c}{ EXEMPLOS } \\
\hline I. Doenças dermatológicas & Dermatite atópica, dermatite de contacto, psoríase, linfoma cutâneo de células T, escabiose \\
\hline II. Doenças sistémicas & $\begin{array}{l}\text { Insuficiência renal crónica, insuficiência hepática, doenças metabólicas (p. ex., diabetes } \\
\text { mellitus), doenças hematoproliferativas (p. ex., policitemia vera), doenças infeciosas, prurido } \\
\text { induzido por medicamentos, linfoma de Hodgkin, tumores sólidos }\end{array}$ \\
\hline III. Doenças neurológicas & $\begin{array}{l}\text { Polineuropatia, síndromes de compressão nervosa (p. ex. prurido braquiorradial ou notalgia } \\
\text { parestética), nevralgia pós-herpética }\end{array}$ \\
\hline $\begin{array}{l}\text { IV. Doenças psiquiátricas e } \\
\text { somatoformes }\end{array}$ & Delírio de parasitose, síndromes depressivos \\
\hline V. Multifactorial & \\
\hline VI. Origem desconhecida & \\
\hline
\end{tabular}




\section{Educação Médica Contínua}

Inúmeros instrumentos têm sido desenvolvidos para a aferição das múltiplas dimensões do PC. A intensidade do prurido pode ser facilmente medida através de escalas monodimensionais como a escala numérica ou a escala analógica visual, semelhante às que são usadas para aferir a dor. A escala categórica da dor constitui uma alternativa, na qual o doente classifica a intensidade do sintoma recorrendo a adjetivos com aumento gradual de intensidade (0 - 4; $0=$ sem prurido, 4 = pior prurido imaginável). Todas estas escalas foram validadas para o uso na avaliação do PC. ${ }^{12}$

Recentemente foram desenvolvidos instrumentos para a aferição da evolução do sintoma ao longo do tempo e a resposta ao tratamento. Em particular revelou-se útil questionar o doente em consultas de seguimento sobre a intensidade do prurido atual em comparação com o início do tratamento, sendo que os resultados podem variar entre $+100 \%$ (melhoria total do nível de prurido) e - $100 \%$ (agravamento absoluto do nível de prurido). Este instrumento (Dynamic Pruritus Score) permite assim perceber rapidamente se o tratamento está a ser bem-sucedido ou se pelo contrário uma mudança de estratégia é necessária. ${ }^{13}$

Outro instrumento de grande utilidade é o índice de benefício do doente (Patient Benefit Index). Antes de se iniciar o tratamento, o doente é convidado a preencher um questionário, composto por 27 itens, sobre a relevância de diversos benefícios terapêuticos. Em consultas de seguimento, após o início do tratamento, pode-se aferir, através de um outro questionário, em que medida estes benefícios terapêuticos foram alcançados, de modo a calcular o índice individualizado de benefício. ${ }^{14}$ Este instrumento permite assim ao médico assistente perceber as preocupações e objectivos do doente e direcionar o tratamento de modo a corresponder a esses objectivos.

Também relevante é a aferição de doenças associadas ao PC. Em particular, depressão e ansiedade são comuns neste grupo de doentes. A escala de depressão e ansiedade hospitalar (Hospital Anxiety and Depression Scale), por exemplo, permite uma célere triagem destes distúrbios. ${ }^{15}$ O impacto da doença prurítica na qualidade de vida deve também ser avaliado, podendo-se recorrer ao questionário ItchyQol (específico para doenças pruríticas) ${ }^{16}$ ou DLQI (Dermatology Life Quality Index), caso haja alterações cutâneas. ${ }^{17}$ Um grupo de dermatologistas europeus especializados em PC considerou prioritário o uso na rotina clínica ${ }^{18}$ de instrumentos avaliando a intensidade do sintoma e o impacto na qualidade de vida.

A categorização dos doentes de acordo com a classificação clínica (ver secção Classificação Clínica e Etiológica) auxilia na escolha dos exames complementares de diagnóstico necessários. Em doentes com dermatoses, biopsias para investigação histológica ou imunofluorescência direta para excluir doenças de origem auto-imune podem ser indicadas. No caso do PC se apresentar sem alteração cutânea, análises clínicas são determinantes para a identificação de possíveis doenças sistémicas subjacentes ( $p$. ex. prurido colestático, nefrogénico, diabetogénico ou subjacente a doença hematológica). Caso o doente se apresente com lesões secundárias crónicas como prurigo ou líquen simples, uma dermatose subjacente nem sempre é clinicamente detetável. Assim sendo, tanto biopsias como uma bateria de análises clínicas de modo a despistar uma possível doença sistémica subjacente podem ser necessárias. Exames imagiológicos estão ainda indicados em caso de PC com menos de um ano de evolução para excluir doença maligna (linfoma ou tumores sólidos) como causa subjacente. Um algoritmo diagnóstico detalhado pode ser consultado nas guidelines europeias sobre o diagnóstico e tratamento de PC. ${ }^{19}$

\section{TRATAMENTO}

Devido à sua multidimensionalidade etiológica e clínica, o tratamento do PC é altamente complexo. Medidas terapêuticas básicas devem ser adoptadas desde logo, mesmo que a causa subjacente à doença prurítica não esteja ainda estabelecida. A xerose cutânea é muito frequente em doentes com PC, devendo ser tratada de forma consequente com emolientes. Em especial, compostos com propriedades antipruríticas como ureia ou polidocanol devem ser recomendados, visto que permitem obter um alívio rápido dos sintomas. Adicionalmente, anti-histamínicos em dose elevada podem ser recomendados independentemente da causa subjacente ao

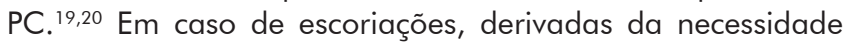
de coçar para obter alívio, corticoesteroides tópicos podem ser introduzidos de forma a acelerar a resolução destas lesões. Todavia, um tratamento prolongado com estes compostos deverá ser evitado devido ao risco de atrofia cutânea. ${ }^{19,20}$ Os inibidores da calcineurina tópicos, tacrolímus e pimecrolimus, constituem uma alternativa para um tratamento prolongado, visto que não causam atrofia cutânea. No entanto, a exposição solar deve ser evitada após a aplicação destes compostos. Em doentes com dermatite atópica, estes agentes podem ser utilizados de forma pró-ativa 3 vezes por semana de forma a evitar o aparecimento do eczema.

Após terminar a investigação diagnóstica, e caso uma causa subjacente ao PC tenha sido encontrada, esta deve ser tratada, se possível. Se por outro lado a origem do PC permanecer desconhecida ou não seja passível de tratamento (p. ex. em caso de doença oncológica terminal ou prurido derivado a polineuropatia) o tratamento do PC deverá ser sintomático.

Diversas terapias sistémicas têm mostrado eficácia no tratamento do PC e devem ser utilizadas consoante a etiologia subjacente. ${ }^{19,20}$ Anti-histamínicos, talvez os agentes sistémicos mais frequentemente utilizados no PC, revelam eficácia no tratamento de doenças pruríticas induzidas por histamina, como é o caso da urticária, mas são ineficazes em doenças mediadas por mecanismos alternativos. ${ }^{21,22}$

Em doenças inflamatórias como a dermatite atópica, que não sejam controladas por agentes tópicos (p. ex. corticoesteroides ou inibidores da calcineurina tópicos) ou por fototerapia, agentes imunossupressores sistémicos devem ser iniciados. Neste grupo destaca-se a ciclosporina A, que diminui a libertação de citocinas pro-inflamatórias ao inibir 
calcineurina e por sua vez a atividade de células T. ${ }^{23} \mathrm{O}$ médico assistente deverá estar atento a possíveis efeitos adversos, como a insuficiência renal, hipertensão e dislipidémia, e monitorizar atentamente o doente. Metotrexato e azatioprina constituem agentes imunossupressores alternativos com aplicação na dermatite atópica. ${ }^{19,20}$

No caso de prurido associado a doenças sistémicas, fármacos anticonvulsivos e antidepressivos têm mostrado eficácia. Gabapentina revela propriedades antipruríticas em doentes com colestase ou insuficiência renal crónica, ${ }^{24}$ enquanto pregabalina pode ser usado para tratar prurido urémico. ${ }^{25}$ Também em doenças de foro neurológico como a polineuropatia diabética ou síndromes compressivos de nervo periférico, agentes anticonvulsivos ou antidepressivos são opções válidas. ${ }^{25-27} \mathrm{Em}$ casos de doença localizada (p. ex. prurido braquiorradial ou notalgia parestética), a aplicação tópica de capsaicina (como creme ou adesivo cutâneo) constitui uma alternativa válida com a vantagem de ter menos efeitos laterais. ${ }^{28}$

Também antidepressivos inibidores seletivos da recaptação de serotonina (p. ex. paroxetina), tricíclicos (p. ex. amitriptilina) e tetracíclicos (p. ex. mirtazapina) têm propriedades antipruríticas, sendo eficazes em diversas indicações nomeadamente em prurido paraneoplásico, ${ }^{29}$ em prurido associado a policitemia vera ou outras doenças mieloproliferativas30 e prurido somatoforme. ${ }^{31}$ Adicionalmente, antidepressivos podem ser associados a anticonvulsivos no tratamento do PC de origem neuropática. ${ }^{32}$

Antagonistas de recetores opioides $\mu$, tanto por via intravenosa (naloxona) como oral (naltrexona), constituem opções terapêuticas para prurido colestático refratário a terapias de primeira linha. ${ }^{33}$ Além disso, alguns dados revelaram igualmente eficácia destes agentes no tratamento de prurido urémico. ${ }^{34}$

Nos dias de hoje a polimedicação observada sobretudo em doentes idosos contribui para o aumento da incidência de PC nesta faixa etária. Múltiplos fármacos podem causar prurido através de diversos mecanismos ( $p$. ex. metabolitos acumulados na pele, aumento da concentração de agentes pruritogénicos, colestase, entre outros). Tipicamente existe uma relação temporal entre a introdução do agente responsável pelo prurido e o início dos sintomas. Estes fármacos deverão ser descontinuados se possível, ou substituídos por um fármaco alternativo de uma classe diferente. Todavia, o prurido pode persistir mesmo após descontinuação do medicamento responsável pelo prurido. Neste caso um tratamento dirigido ao mecanismo patológico subjacente será necessário. ${ }^{35}$

Por vezes, após minuciosa investigação etiológica, a origem do PC permanece desconhecida. Nestes casos recomenda-se iniciar o tratamento com anti-histamínicos. Caso estes agentes não surtam o efeito desejado, anticonvulsivos em monoterapia ou em combinação com antidepressivos constituem o passo seguinte. Caso o PC se mostre refractário a estes tratamentos, recomenda-se como alternativa a fototerapia (UVB-31 1nm) ou uma terapia com naltrexona. ${ }^{20}$
Além do tratamento do prurido em si, condições associadas, como distúrbios de sono ou ansiedade e síndromes depressivos, não devem ser negligenciadas. Nestas circunstâncias, é aconselhado discutir com o doente o acompanhamento por um especialista da área como por exemplo um psiquiatra no caso de doença mental concomitante. Além disso alguns doentes desenvolvem um comportamento automatizado, coçando-se mesmo na ausência de prurido. Técnicas de relaxamento e gestão de stress podem ser benéficas para estes doentes. ${ }^{36,37}$

\section{NOVOS FÁRMACOS}

Nos últimos anos têm-se assistido ao desenvolvimento de novos fármacos para o tratamento do PC bem como de dermatites pruríticas. ${ }^{38}$ Antagonistas de recetores da neuroquinina 1 , nomeadamente o aprepitant, têm merecido destaque. Este medicamento, indicado para a prevenção de náusea e vómito no pós-operatório, revelou efeitos antipruríticos. ${ }^{39} \mathrm{Em}$ particular, o prurido associado a dermatite atópica, linfoma cutâneo de células T e prurigo crónico responde ao tratamento com aprepitant. ${ }^{40}$ Outros compostos pertencentes a esta família, em desenvolvimento para tratamento do PC, incluem serlopitant, tradipitant e orvepitant.

Dupilumab, um anticorpo anti-interleucina 4/13, levou a uma melhoria significativa tanto do eczema como do prurido num ensaio clínico com doentes com dermatite atópica. ${ }^{41}$ Também antagonistas da interleucina-31 (nemolizumab) mostraram eficácia no tratamento de prurido associado a doenças inflamatórias. No tratamento da dermatite atópica o uso destes fármacos induziu uma rápida melhoria do prurido e permitiu diminuir o uso de corticoesteróides tópicos. ${ }^{42}$ Inibidores seletivos das Janus quinases (tofacitinib) ${ }^{43,44}$ assim como inibidores da fosfodiesterase- 4 (crisaborole) ${ }^{45}$ são outros agentes recentemente desenvolvidos para o tratamento de prurido associado a dermatites.

Agonistas de receptores opioides $\mathrm{x}$ são agentes promissores, que atuam a nível espinal. Nalfurafina, já aprovado no Japão, mostrou efeitos antipruríticos em doentes com insuficiência renal crónica necessitando de hemodiálise. ${ }^{46}$ Também nalbufina, um agente simultaneamente antagonista de recetores opioides $\mu$ e agonista de recetores opioides $\mathrm{K}$, mostrou eficácia antiprurítica em doentes sujeitos a hemodiálise. ${ }^{47}$

Para o tratamento de prurido colestático, causado pela acumulação de agentes pruríticos como ácidos biliares, ${ }^{48}$ autotaxina e opioides endógenos entre outros, sequestradores de ácidos biliares (p. ex. colesevelam) têm sido testados. Todavia estes agentes falharam em mostrar superioridade relativamente ao tratamento com placebo. ${ }^{49}$ Inibidores de transportadores de ácidos biliares representam uma possível alternativa, estando a decorrer ensaios clínicos em doentes com cirrose biliar. ${ }^{50}$

\section{CONCLUSÃO}

PC é uma condição médica altamente complexa devido à sua multidimensionalidade etiológica e clínica. Na abordagem 


\section{Educação Médica Contínua}

ao doente com PC, é essencial proceder a uma procura sistemática da origem do prurido através de uma anamnese e exame objectivo completos, bem como de exames complementares de diagnóstico direcionados. O tratamento do PC permanece um desafio, sendo que a administração sistémica de fármacos é frequentemente necessária. Um maior conhecimento sobre os mecanismos subjacentes ao PC permitirá o desenvolvimento de fármacos mais eficazes.

Conflitos de interesse: Os autores declaram não possuir conflitos de interesse.

Suporte financeiro: $O$ presente trabalho não foi suportado por nenhum subsídio ou bolsa.

Conflicts of interest: The authors have no conflicts of interest to declare.

Financing Support: This work has not received any contribution, grant or scholarship.

\section{REFERÊNCIAS}

1. Stander S, Schafer I, Phan NQ, Blome C, Herberger K, Heigel $\mathrm{H}$, et al. Prevalence of chronic pruritus in Germany: results of a cross-sectional study in a sample working population of 11,730. Dermatology. 2010; 221:229-35.

2. Matterne U, Apfelbacher CJ, Loerbroks A, Schwarzer $T$, Buttner $M$, Ofenloch $R$, et al. Prevalence, correlates and characteristics of chronic pruritus: a population-based cross-sectional study. Acta Derm Venereol. 2011; 91:674-9.

3. Kopyciok ME, Stander HF, Osada N, Steinke S, Stander S. Prevalence and Characteristics of Pruritus: A One-Week Cross-sectional Study in a German Dermatology Practice. Acta Derm Venereol. 2016; 96:50-5.

4. Hay RJ, Johns NE, Williams HC, Bolliger IW, Dellavalle RP, Margolis DJ, et al. The global burden of skin disease in 2010: an analysis of the prevalence and impact of skin conditions. J Invest Dermatol. 2014; 134:1527-34.

5. Stander S, Pogatzki-Zahn E, Stumpf A, Fritz F, Pfleiderer $B$, Ritzkat $A$, et al. Facing the challenges of chronic pruritus: a report from a multi-disciplinary medical itch centre in Germany. Acta Derm Venereol. 2015; 95:266-71.

6. Dhand A, Aminoff MJ. The neurology of itch. Brain. 2014; 137: 313-22.

7. Liu T, Ji RR. New insights into the mechanisms of itch: are pain and itch controlled by distinct mechanisms? Pflugers Arch. 2013; 465:1671-85.

8. Johanek LM, Meyer RA, Friedman RM, Greenquist KW, Shim B, Borzan J, et al. A role for polymodal C-fiber afferents in nonhistaminergic itch. J Neurosci. 2008; 28:7659-69.

9. Stander S, Weisshaar E, Mettang T, Szepietowski JC, Carstens E, Ikoma A, et al. Clinical classification of itch: a position paper of the International Forum for the Study of Itch. Acta Derm Venereol. 2007;87:291-4.

10. Weisshaar E, Gieler U, Kupfer J, Furue M, Saeki H,
Yosipovitch G. Questionnaires to assess chronic itch: a consensus paper of the special interest group of the International Forum on the Study of Itch. Acta Derm Venereol. 2012; 92:493-6.

11. Yosipovitch G, Zucker I, Boner G, Gafter U, Shapira Y, David M. A questionnaire for the assessment of pruritus: validation in uremic patients. Acta Derm Venereol. 2001; 81:108-11.

12. Phan NQ, Blome C, Fritz F, Gerss J, Reich A, Ebata T, et al. Assessment of pruritus intensity: prospective study on validity and reliability of the visual analogue scale, numerical rating scale and verbal rating scale in 471 patients with chronic pruritus. Acta Derm Venereol. 2012; 92:502-7.

13. Stander S, Blome C, Anastasiadou Z, Zeidler C, Jung KA, Tsianakas A, et al. Dynamic Pruritus Score: Evaluation of the Validity and Reliability of a New Instrument to Assess the Course of Pruritus. Acta Derm Venereol. 2017; 97:230-4.

14. Augustin M, Radtke MA, Zschocke I, Blome C, Behechtnejad J, Schafer I, et al. The patient benefit index: a novel approach in patient-defined outcomes measurement for skin diseases. Arch Dermatol Res. 2009; 301:561-71.

15. Snaith RP. The Hospital Anxiety And Depression Scale. Health Qual Life Outcomes. 2003; 1: 29.

16. Desai NS, Poindexter GB, Monthrope YM, Bendeck SE, Swerlick RA, Chen SC. A pilot quality-of-life instrument for pruritus. J Am Acad Dermatol. 2008; 59:234-44.

17. Finlay AY, Khan GK. Dermatology Life Quality Index (DLQI)--a simple practical measure for routine clinical use. Clin Exp Dermatol. 1994; 19:210-6.

18. Stander S, Zeidler C, Riepe C, Steinke S, Fritz F, Bruland $P$, et al. European EADV network on assessment of severity and burden of Pruritus (PruNet): first meeting on outcome tools. J Eur Acad Dermatol Venereol. 2016; 30:1144-7.

19. Weisshaar E, Szepietowski JC, Darsow U, Misery L, Wallengren J, Mettang T, et al. European guideline on chronic pruritus. Acta Derm Venereol. 2012; 92:563-81.

20. Ständer S, Zeidler C, Augustin M, Bayer G, Kremer A, Legat FJ, et al. S2k-Leitlinie zur Diagnostik und Therapie des chronischen Pruritus. J Dtsch Dermatol Ges. 2017; 15:860-73.

21. Leslie TA, Greaves MW, Yosipovitch G. Current topical and systemic therapies for itch. Handb Exp Pharmacol. 2015; 226:337-56.

22. Stander S, Weisshaar E, Luger TA. Neurophysiological and neurochemical basis of modern pruritus treatment. Exp Dermatol. 2008; 17:161-9.

23. Matsuda S, Koyasu S. Mechanisms of action of cyclosporine. Immunopharmacology. 2000; 47:119-25.

24. Gunal AI, Ozalp G, Yoldas TK, Gunal SY, Kirciman E, Celiker H. Gabapentin therapy for pruritus in haemodialysis patients: a randomized, placebo-controlled, double-blind trial. Nephrol Dial Transplant. 2004; 19:3137-9. 


\section{Educação Médica Contínua}

25. Ehrchen J, Stander S. Pregabalin in the treatment of chronic pruritus. J Am Acad Dermatol. 2008; 58:S36-7.

26. Winhoven SM, Coulson IH, Bottomley WW. Brachioradial pruritus: response to treatment with gabapentin. $\mathrm{Br}$ J Dermatol. 2004; 150:786-7.

27. Callaghan BC, Cheng HT, Stables CL, Smith AL, Feldman EL. Diabetic neuropathy: clinical manifestations and current treatments. Lancet Neurol. 2012; 11:521-34.

28. Steinke S, Gutknecht M, Zeidler C, Dieckhofer AM, Herrlein $\mathrm{O}$, Luling $\mathrm{H}$, et al. Cost-effectiveness of an $8 \% \mathrm{Cap}$ saicin Patch in the Treatment of Brachioradial Pruritus and Notalgia Paraesthetica, Two Forms of Neuropathic Pruritus. Acta Derm Venereol. 2017; 96:71-6.

29. Zylicz Z, Smits C, Krajnik M. Paroxetine for pruritus in advanced cancer. J Pain Symptom Manage. 1998; 16:121-4.

30. Tefferi A, Fonseca R. Selective serotonin reuptake inhibitors are effective in the treatment of polycythemia vera-associated pruritus. Blood. 2002; 99:2627.

31. Biondi M, Arcangeli T, Petrucci RM. Paroxetine in a case of psychogenic pruritus and neurotic excoriations. Psychother Psychosom. 2000; 69:165-6.

32. Pereira MP, Kremer AE, Mettang T, Stander S. Chronic Pruritus in the Absence of Skin Disease: Pathophysiology, Diagnosis and Treatment. Am J Clin Dermatol. 2016; 17:337-48.

33. Kremer AE, Namer B, Bolier R, Fischer MJ, Oude Elferink RP, Beuers U. Pathogenesis and Management of Pruritus in PBC and PSC. Dig Dis. 2015; 33 Suppl 2:164-75.

34. Peer G, Kivity S, Agami O, Fireman E, Silverberg D, Blum $M$, et al. Randomised crossover trial of naltrexone in uraemic pruritus. Lancet. 1996; 348:1552-4.

35. Reich A, Stander S, Szepietowski JC. Drug-induced pruritus: a review. Acta Derm Venereol. 2009; 89:236-44.

36. Bathe A, Matterne U, Dewald M, Grande T, Weisshaar E. Educational multidisciplinary training programme for patients with chronic pruritus. Acta Derm Venereol. 2009; 89:498-501.

37. Evers AW, Duller P, de Jong EM, Otero ME, Verhaak CM, van der Valk PG, et al. Effectiveness of a multidisciplinary itch-coping training programme in adults with atopic dermatitis. Acta Derm Venereol. 2009; 89:57-63.

38. Pereira MP, Stander S. Chronic Pruritus: Current and Emerging Treatment Options. Drugs. 2017; 77:9991007.

39. Stander S, Siepmann D, Herrgott I, Sunderkotter C, Luger TA. Targeting the neurokinin receptor 1 with aprepitant: a novel antipruritic strategy. PLoS One. 2010; 5:e10968.

40. Stander S, Luger TA. NK-1 Antagonists and Itch. Handb
Exp Pharmacol. 2015; 226:237-55.

41. Beck LA, Thaci D, Hamilton JD, Graham NM, Bieber T, Rocklin R, et al. Dupilumab treatment in adults with moderate-to-severe atopic dermatitis. N Engl J Med. 2014; 371:130-9.

42. Nemoto $O$, Furue $M$, Nakagawa $H$, Shiramoto $M$, Hanada $R$, Matsuki $S$, et al. The first trial of CIM331, a humanized anti-human IL-31 receptor A antibody, for healthy volunteers and patients with atopic dermatitis to evaluate safety, tolerability and pharmacokinetics of a single dose in a randomised, double-blind, placebo-controlled study. Br J Dermatol. 2016; 174:296-304.

43. Feldman SR, Thaci D, Gooderham M, Augustin M, de la Cruz C, Mallbris L, et al. Tofacitinib improves pruritus and health-related quality of life up to 52 weeks: Results from 2 randomized phase III trials in patients with moderate to severe plaque psoriasis. J Am Acad Dermatol. 2016;75: 1162-70.e3.

44. Bissonnette R, Papp KA, Poulin Y, Gooderham M, Raman $M$, Mallbris L, et al. Topical tofacitinib for atopic dermatitis: a phase lla randomized trial. Br J Dermatol. 2016; 175:902-11.

45. Paller AS, Tom WL, Lebwohl MG, Blumenthal RL, Boguniewicz $M$, Call RS, et al. Efficacy and safety of crisaborole ointment, a novel, nonsteroidal phosphodiesterase 4 (PDE4) inhibitor for the topical treatment of atopic dermatitis (AD) in children and adults. J Am Acad Dermatol. 2016; 75:494-503.e4.

46. Wikstrom B, Gellert R, Ladefoged SD, Danda Y, Akai $M$, Ide $K$, et al. Kappa-opioid system in uremic pruritus: multicenter, randomized, double-blind, placebo-controlled clinical studies. J Am Soc Nephrol. 2005; 16:3742-7.

47. Hawi A, Alcorn H, Jr., Berg J, Hines C, Hait H, Sciascia T. Pharmacokinetics of nalbuphine hydrochloride extended release tablets in hemodialysis patients with exploratory effect on pruritus. BMC Nephrol. 2015; 16:47.

48. Beuers U, Kremer AE, Bolier R, Elferink RP. Pruritus in cholestasis: facts and fiction. Hepatology. 2014; 60:399407.

49. Kuiper EM, van Erpecum KJ, Beuers U, Hansen BE, Thio $H B$, de Man RA, et al. The potent bile acid sequestrant colesevelam is not effective in cholestatic pruritus: results of a double-blind, randomized, placebo-controlled trial. Hepatology. 2010; 52:1334-40.

50. Hegade VS, Kendrick SF, Dobbins RL, Miller SR, Richards D, Storey J, et al. BAT1 17213: Ileal bile acid transporter (IBAT) inhibition as a treatment for pruritus in primary biliary cirrhosis: study protocol for a randomised controlled trial. BMC Gastroenterol. 2016; 16:71. 


\section{Educação Médica Contínua}

\section{VERIFIQUE O QUE APRENDEU}

1. Qual destas afirmações é correta?

a) Estima-se que a prevalência de prurido crónico na população seja de cerca de $5 \%$.

b) A qualidade de vida está frequentemente afectada em doentes com prurido crónico e deve ser investigada.

c) Doentes com prurido crónico apresentam sempre lesões cutâneas que devem ser investigadas por histologia.

d) Prurido crónico define-se como prurido com duração igual ou superior a 6 meses.

e) Todos os doentes com prurido crónico devem ser referenciados a um psiquiatra para despiste de doença mental.

2. Qual destas afirmações sobre a fisiopatologia do prurido crónico é correta:

a) Fibras nervosas do tipo $A \beta$ desempenham um papel central na transmissão de sinais pruríticos.

b) Estudos de ressonância magnética funcional revelaram um padrão de ativação neuronal comum às diversas doenças pruríticas.

c) A sensibilização nervosa central e periférica contribui para a cronificação do sintoma.

d) Fibras nervosas do tipo A $\delta$ não estão envolvidas na transmissão periférica do prurido.

e) Fibras nervosas do tipo $C$ insensíveis a histamina transmitem dor mas não prurido.

3. Qual destas categorias pertence à classificação clínica proposta pelo IFSI?

a) Doentes com doença cutânea inflamatória

b) Doentes sem alteração cutânea

c) Doentes com lesões crónicas (prurigo crónico e/ou líquen simples crónico)

d) Todas as hipóteses são verdadeiras

e) Todas as hipóteses são falsas.

4. Qual destas doenças não está associada ao prurido crónico?

a) Linfoma de células $T$

b) Linfoma de Hodgkin

c) Hepatite $\mathrm{C}$

d) $\mathrm{VIH}$

e) Todas as hipóteses acima mencionadas podem estar associadas ao prurido crónico.

5. Qual dos seguintes aspectos não pertence à anamnese específica do prurido crónico:

a) Co-medicação

b) Distúrbios de sono c) Sintomas de depressão e ansiedade

d) Duração do prurido

e) Flutuação diária da intensidade do prurido

6. Qual dos seguintes procedimentos de diagnóstico não é habitualmente efectuado em doentes com prurido crónico?

a) Consulta de otorrinolaringologia

b) Sonografia abdominal

c) Inspeção e exame objectivo

d) Radiografia torácica

e) Análises clínicas

7. Qual das seguintes doenças ocorre frequentemente como consequência do prurido crónico?

a) Hipertensão arterial

b) Insuficiência renal crónica

c) Síndrome do intestino irritável

d) Distúrbios de sono

e) Síndrome do túnel cárpico

8. No tratamento do prurido crónico:

a) Agentes sistémicos não têm indicação no prurido associado a dermatites.

b) Emolientes são geralmente insuficientes no tratamento do prurido crónico e devem ser desaconselhados.

c) Agentes tópicos são geralmente suficientes.

d) Antidepressivos só devem ser iniciados em caso de síndrome depressivo concomitante.

e) Deve-se iniciar o tratamento antes da causa subjacente ter sido estabelecida.

9. Qual das seguintes associações etiologia/tratamento é correta?

a) Dermatite atópica - capsaicina

b) Prurido urémico - rifampicina

c) Prurido aquagénico - pregabalina

d) Urticária - fluoxetina

e) Nevralgia pós-herpética - cetirizina

10. Qual destas classes de fármacos não está a ser testada para o tratamento do prurido crónico?
a) Anticorpo monoclonal anti-interleucina-31
b) Inibidores da enzima conversora da angiotensina
c) Antagonistas de receptores da neuroquinina 1
d) Inibidores da fosfodiesterase-4
e) Inibidores de transportadores de ácidos biliares 